\title{
A study of optimization for Steffensen-type methods with frozen divided differences
}

\author{
J. A. Ezquerro ${ }^{a}$, M. Grau-Sánchez ${ }^{b}$, M. A. Hernández-Verón ${ }^{a}$, M. Noguera ${ }^{b}$ \\ a Dpt. of Mathematics and Computation, University of La Rioja \\ 26004 Logroño, Spain \\ E-mail address: <jezquer ><mahernan>@unirioja.es \\ b Dpt. of Applied Mathematics II, Technical University of Catalonia \\ 08034 Barcelona, Spain \\ E-mail address: <miquel.grau><miquel.noguera>@upc.edu
}

\begin{abstract}
A local convergence analysis for a generalization of a family of Steffensen-type iterative methods with three frozen steps is presented for solving nonlinear equations. From the use of three classical divided difference operators, we study four families of iterative methods with optimal local order of convergence. Then, new variants of the family of iterative methods is constructed, where a study of the computational efficiency is carried out. Moreover, the semilocal convergence for these families is also studied. Finally, an application of nonlinear integral equations of mixed Hammerstein type is presented, where multiple precision and a stopping criterion are implemented without using any known root. In addition, a study, where we compare orders, efficiencies and elapsed times of the methods suggested, supports the theoretical results obtained.
\end{abstract}

Keywords: Divided difference, order of convergence, efficiency, system of nonlinear equations, iterative methods.

Mathematics Subject Classification: 65H10, 65Y20

\section{Introduction}

Let $F: D \subset \mathbb{R}^{m} \longrightarrow \mathbb{R}^{m}$ and assume that $F$ has, at least, third-order Fréchet derivatives with continuity on a convex nonempty set $D$. Suppose that the equation

$$
F(x)=0
$$

has a solution $x^{*} \in D$ at which $F^{\prime}\left(x^{*}\right)$ is nonsingular. In this study we are concerned with the local convergence and the computational efficiency of a family of iterative methods for solving (1). We analyze the following iteration family

$$
\Phi:\left\{\begin{array}{l}
x \in D \subset \mathbb{R}^{m}, \\
y=x-\Theta^{-1} F(x), \\
z=y-\Theta^{-1}\left(\alpha_{1} F(x)+\alpha_{2} F(y)\right), \\
X=z-\Theta^{-1}\left(\beta_{1} F(x)+\beta_{2} F(y)+\beta_{3} F(z)\right),
\end{array}\right.
$$

where $\alpha_{1}, \alpha_{2}, \beta_{1}, \beta_{2}, \beta_{3}$ are real parameters and $\Theta$ can be the following three different divided difference operators:

$$
\Theta_{1}=[x, x+F(x) ; F], \quad \Theta_{2}=[x-F(x), x ; F] \quad \text { or } \quad \Theta_{3}=[x-F(x), x+F(x) ; F]
$$


which give three variants of the Steffensen method [16] if we only consider the first step of five-parameter iteration family $\Phi$ defined in (2); namely, $X=x-\Theta^{-1} F(x)$. Observe in (2) that the same operator $\Theta$ is used in the three steps in order to minimize the computational cost. Several papers in the recent literature taking into account iterative methods with an operator repeated, that we call frozen, can be found in $[1-4,12]$.

We give a more general expression of the first order divided difference operator setting $\varepsilon_{A}=A-x^{*}$ and $\varepsilon_{B}=B-x^{*}$, where $x^{*} \in \mathbb{R}^{m}$ is a simple solution of nonlinear equation (1), and $A$ and $B$ are functions of $x$ with $A\left(x^{*}\right)=x^{*}$ and $B\left(x^{*}\right)=x^{*}$. From papers $[9,10]$, the divided difference operator, in terms of the errors $\varepsilon_{A}$ and $\varepsilon_{B}$, can be expressed by

$$
[A, B ; F]=\Gamma\left(I+A_{2}\left(\varepsilon_{A}+\varepsilon_{B}\right)+A_{3}\left(\varepsilon_{A}^{2}+\varepsilon_{A} \varepsilon_{B}+\varepsilon_{B}^{2}\right)+O_{3}\left(\varepsilon_{A}, \varepsilon_{B}\right)\right),
$$

where $\varepsilon^{p}=(\varepsilon, \stackrel{p}{\cdots}, \varepsilon)$. Moreover, $O_{3}\left(\varepsilon_{A}, \varepsilon_{B}\right)$ is $O\left(\varepsilon_{A}^{p} \varepsilon_{B}^{q}\right)$ with $0 \leq p, q \leq 3$ and such that $p+q=3$, and $A_{k}=\Gamma^{-1} F^{(k)}\left(x^{*}\right) / k ! \in \mathcal{L}_{k}\left(\mathbb{R}^{m}, \mathbb{R}^{m}\right), k \geq 2$, with $\Gamma=F^{(1)}\left(x^{*}\right)$. From (4) and considering the expression of $F(x)$ in powers of $e=x-x^{*}$ :

we obtain

$$
F(x)=\Gamma\left(e+A_{2} e^{2}+A_{3} e^{3}+O_{4}(e)\right)
$$

$$
\begin{aligned}
\Theta_{1} & =[x, x+F(x) ; F]=\Gamma\left(I+A_{2}(2 e+\widetilde{e})+A_{3}\left(3 e^{2}+3 e \widetilde{e}+\widetilde{e}^{2}\right)+A_{2} \Gamma A_{2} e^{2}+O_{3}(e, \widetilde{e})\right), \\
\Theta_{2} & =[x-F(x), x ; F]=\Gamma\left(I+A_{2}(2 e-\widetilde{e})+A_{3}\left(3 e^{2}-3 e \widetilde{e}+\widetilde{e}^{2}\right)-A_{2} \Gamma A_{2} e^{2}+O_{3}(e, \widetilde{e})\right), \\
\Theta_{3} & =[x-F(x), x+F(x) ; F]=\Gamma\left(I+2 A_{2} e+A_{3}\left(3 e^{2}+\widetilde{e}^{2}\right)+O_{3}(e, \widetilde{e})\right),
\end{aligned}
$$

where $\widetilde{e}=\Gamma e$. From (6)-(8), the following respective inverse operators are

$$
\begin{aligned}
\Theta_{1}^{-1}= & \left(I-A_{2}(2 e+\widetilde{e})-A_{3}\left(3 e^{2}+3 e \widetilde{e}+\widetilde{e}^{2}\right)-A_{2} \Gamma A_{2} e^{2}+4\left(A_{2} e\right)^{2}\right. \\
& \left.+2\left(A_{2} e A_{2} \widetilde{e}+A_{2} \widetilde{e} A_{2} e\right)+\left(A_{2} \widetilde{e}\right)^{2}+O_{3}(e, \widetilde{e})\right) \Gamma^{-1}, \\
\Theta_{2}^{-1}= & \left(I-A_{2}(2 e-\widetilde{e})-A_{3}\left(3 e^{2}-3 e \widetilde{e}+\widetilde{e}^{2}\right)+A_{2} \Gamma A_{2} e^{2}+4\left(A_{2} e\right)^{2}\right. \\
& \left.-2\left(A_{2} e A_{2} \widetilde{e}+A_{2} \widetilde{e} A_{2} e\right)+\left(A_{2} \widetilde{e}\right)^{2}+O_{3}(e, \widetilde{e})\right) \Gamma^{-1}, \\
\Theta_{3}^{-1}= & \left(I-2 A_{2} e-A_{3}\left(3 e^{2}+\widetilde{e}^{2}\right)+4\left(A_{2} e\right)^{2}+O_{3}(e, \widetilde{e})\right) \Gamma^{-1} .
\end{aligned}
$$

Using the operators defined in (3), the first step of the family of iteration functions $\Phi$ defined in (2) gives the following three possibilities

$$
\begin{aligned}
& y^{+}=x-[x, x+F(x) ; F]^{-1} F(x), \\
& y^{-}=x-[x-F(x), x ; F]^{-1} F(x), \\
& y^{\mp}=x-[x-F(x), x+F(x) ; F]^{-1} F(x) .
\end{aligned}
$$

Subtracting $x^{*}$ from both sides of the preceding equations and taking into accoutn (9)-(11) and (5), we have the following respective errors

$$
\begin{aligned}
& e_{y}^{+}=y^{+}-x^{*}=A_{2}(e+\widetilde{e}) e-A_{2}(2 e+\widetilde{e}) A_{2}(e+\widetilde{e}) e+A_{3}\left(2 e^{2}+3 e \widetilde{e}+\widetilde{e}^{2}\right) e+A_{2} \Gamma A_{2} e^{3}+O_{4}(e, \widetilde{e}), \\
& e_{y}^{-}=y^{-}-x^{*}=A_{2}(e-\widetilde{e}) e-A_{2}(2 e-\widetilde{e}) A_{2}(e-\widetilde{e}) e+A_{3}\left(2 e^{2}-3 e \widetilde{e}+\widetilde{e}^{2}\right) e-A_{2} \Gamma A_{2} e^{3}+O_{4}(e, \widetilde{e}), \\
& e_{y}^{\mp}=y^{\mp}-x^{*}=A_{2} e^{2}-2\left(A_{2} e\right)^{2} e+A_{3}\left(2 e^{2}+\widetilde{e}^{2}\right) e+O_{4}(e, \widetilde{e}) .
\end{aligned}
$$

So, we conclude the well-known result that the three iterative methods presented from considering the first step of (2) using the operators defined in (3) have at least quadratic convergence.

In order to develop, analyze and study the error expressions of $X-x^{*}$, we begin in the scalar case that is easier to do symbolic manipulations. The results in the one-dimensional case will be generalized to $m$-dimensional case regarding the set of values of the parameters that improve the local order of convergence. If we want to approximate a root $x^{*} \in \mathbb{R}$ of a scalar nonlinear equation $f(x)=0$, where $f: I \subset \mathbb{R} \longrightarrow \mathbb{R}$ is a nonlinear function and $I$ a nonempty neighborhood of $x^{*}$, we then define 


$$
\phi:\left\{\begin{array}{l}
x \in I \subset \mathbb{R} \\
y=x-\theta^{-1} f(x) \\
z=y-\theta^{-1}\left(\alpha_{1} f(x)+\alpha_{2} f(y)\right) \\
X=z-\theta^{-1}\left(\beta_{1} f(x)+\beta_{2} f(y)+\beta_{3} f(z)\right),
\end{array}\right.
$$

where the operator $\theta$ is now the scalar divided difference defined in an unique way by the expression

$$
\theta=[u, v ; f]=\frac{f(v)-f(u)}{v-u} .
$$

In (15), the scalar iteration family $\phi$, given in (15), uses divided difference $\theta$ which can be

$$
\theta_{1}=[x, x+f(x) ; f], \quad \theta_{2}=[x-f(x), x ; f] \quad \text { or } \quad \theta_{3}=[x-f(x), x+f(x) ; f] .
$$

In the scalar case, the expressions of the inverse operators (9)-(11) and the errors (12)-(14) are easier because we consider the scalar divided difference $\theta \in \mathbb{R}$ instead of the operator $\Theta \in \mathfrak{L}\left(\mathbb{R}^{m}, \mathbb{R}^{m}\right)$ and the commutativity between them allows us an easier symbolic computation.

Once we have done the one-dimensional study, we set the parameters $\alpha_{1}, \alpha_{2}, \beta_{1}, \beta_{2}$ and $\beta_{3}$ for the optimal cases of family (2) according to the local order and the efficiency index. Next, in Section 3, we do a multidimensional study, where we definitely set the methods of family (2) that are optimal in terms of the local order. After that, in Section 4, we analyse the efficiency of the methods of family (2) that we have selected previously from the local order. Then, in Section 5, we analyse the semilocal convergence of the optimal iterative methods previously obtained. Finally, in Section 6, an application of nonlinear integral equations of mixed Hammerstein type is presented, where multiple precision and a stopping criterion are implemented without using any known root. In addition, a study, where we compare orders, efficiencies and elapsed times of the methods suggested, supports the theoretical results obtained.

\section{Maximum local order of convergence in scalar case}

We present in detail the results of the development in order to express the error of family $\phi$ defined in (15) and compute the local error for operators $\theta_{1}$ or $\theta_{2}$ which give the same equations. For operator $\theta_{3}$, it will be done in an analogous way. Developing the error of iteration function $\phi$ with $\theta_{1}$ or $\theta_{2}, e_{n+1}=X-x^{*}$, in powers of $e_{n}=e=x-x^{*}$ until the third order and cancelling the coefficients of the terms up to the second order, we have:

- coefficients of $e$ :

$$
\alpha_{1}+\beta_{1}-\alpha_{1} \beta_{3}=0
$$

- coefficients of $A_{2} e^{2}$ :

$$
\begin{array}{r}
-1-\alpha_{1}+\alpha_{2}-\beta_{1}+\beta_{2}+\beta_{3}+\left(3 \alpha_{1}+\alpha_{1}^{2}-\alpha_{2}\right) \beta_{3}=0, \\
-1-\alpha_{1}+\alpha_{2}-\beta_{1}+\beta_{2}+\beta_{3}+\left(2 \alpha_{1}-\alpha_{2}\right) \beta_{3}=0 .
\end{array}
$$

- coefficients of $A_{2} e \widetilde{e}$ :

In addition, we get three sets of solutions, but only one of them has fourth order. These three families of iterative methods are called $\mathcal{A}, \mathcal{B}$ and $\mathcal{C}$ and they are defined by

$$
\begin{aligned}
& \mathcal{A}=\left\{\alpha_{1}=\alpha, \alpha_{2}=\beta, \beta_{1}=-\alpha, \beta_{2}=1-\beta, \beta_{3}=0\right\}, \\
& \mathcal{B}=\left\{\alpha_{1}=0, \alpha_{2}=\alpha, \beta_{1}=0, \beta_{2}=(1-\alpha)(1-\beta), \beta_{3}=\beta\right\}, \\
& \mathcal{C}=\left\{\alpha_{1}=-1, \alpha_{2}=\alpha, \beta_{1}=-\beta, \beta_{2}=1-\alpha+\alpha \beta, \beta_{3}=\beta\right\} .
\end{aligned}
$$

Summarizing, for $\theta=\theta_{1}$ or $\theta=\theta_{2}$, the iteration family $\phi$ defined in (15) has three members of methods of third order, where the first step of them is $y=x-\theta^{-1} f(x)$ and they are described and analyzed in the next sections. 


\section{$2.1 \quad$ First family}

The first family of iterative methods with order of convergence at least three is:

$$
\phi_{\mathcal{A}}:\left\{\begin{aligned}
y & =x-\theta^{-1} f(x), \\
z & =y-\theta^{-1}(\alpha f(x)+\beta f(y)), \\
X & =z-\theta^{-1}(-\alpha f(x)+(1-\beta) f(y))=\frac{1}{\beta}(\alpha x+(\beta-1-\alpha) y+z), \quad \text { where } \beta \neq 0 .
\end{aligned}\right.
$$

Note that $\phi_{\mathcal{A}}$, defined in (16), is a family of two-step Steffensen-type method which has the following error equation

$$
\left|e_{n+1}\right|= \begin{cases}A_{2}^{2}|(2 e+\widetilde{e})(e+\widetilde{e}) e|+O_{4}(e, \widetilde{e}) & \text { if } \theta=\theta_{1}, \\ A_{2}^{2}|(2 e-\widetilde{e})(e-\widetilde{e}) e|+O_{4}(e, \widetilde{e}) & \text { if } \theta=\theta_{2} .\end{cases}
$$

In both cases, the local order of convergence is at least three and is independent of the real parameters $\alpha$ and $\beta$.

Using the comparative parameter called efficiency index presented by Ostrowski in [13], $E I=\rho^{1 / \omega}$, where $\rho$ is the local order of convergence of the method and $\omega$ represents the number of the evaluations of functions necessary to apply the method per iteration, we obtain that the efficiency index for the family $\phi_{\mathcal{A}}$ given in (16) is $E I_{\mathcal{A}}=3^{1 / 3}=1.442$. An important classical known method of family (16) arises for $\alpha=0$ and $\beta=1$, which is called frozen two-step Steffensen method of third order and given by

$$
\phi_{\mathcal{A}(\alpha=0, \beta=1)}:\left\{\begin{aligned}
y & =x-\theta^{-1} f(x), \\
X & =y-\theta^{-1} f(y) .
\end{aligned}\right.
$$

\subsection{Second family}

The second family of iterative methods is:

$$
\phi_{\mathcal{B}}:\left\{\begin{aligned}
y & =x-\theta^{-1} f(x), \\
z & =y-\alpha \theta^{-1} f(y), \\
X & =z-\theta^{-1}((1-\alpha)(1-\beta) f(y)+\beta f(z)),
\end{aligned}\right.
$$

whose error equation is:

$$
\left|e_{n+1}\right|= \begin{cases}|1-\alpha \beta| A_{2}^{2}|(2 e+\widetilde{e})(e+\widetilde{e}) e|+O_{4}(e, \widetilde{e}) & \text { if } \theta=\theta_{1}, \\ |1-\alpha \beta| A_{2}^{2}|(2 e-\widetilde{e})(e-\widetilde{e}) e|+O_{4}(e, \widetilde{e}) & \text { if } \theta=\theta_{2} .\end{cases}
$$

In general, the order of convergence is at least three, but we have, for $\beta=\frac{1}{\alpha}$ with $\alpha \neq 0$, the following family of iterative methods with order of convergence at least four:

$$
\phi_{\mathcal{B}(\beta=1 / \alpha)}:\left\{\begin{aligned}
y & =x-\theta^{-1} f(x) \\
z & =y-\alpha \theta^{-1} f(x) \\
X & =z-\frac{1}{\alpha} \theta^{-1}\left(-(1-\alpha)^{2} f(y)+f(z)\right) .
\end{aligned}\right.
$$

The three-step iterative method $\phi_{\mathcal{B}(\beta=1 / \alpha)}$ has the following error equation

$$
\left|e_{n+1}\right|= \begin{cases}\left|A_{2}^{3}\right||(e+\widetilde{e}) e|\left|(5-\alpha)(e+\widetilde{e}) e+\widetilde{e}^{2}\right|+O_{5}(e, \widetilde{e}) & \text { for } \theta=\theta_{1}, \\ \left|A_{2}^{3}\right||(e-\widetilde{e}) e|\left|(5-\alpha)(e-\widetilde{e}) e+\widetilde{e}^{2}\right|+O_{5}(e, \widetilde{e}) & \text { for } \theta=\theta_{2} .\end{cases}
$$


In particular, taking $\alpha=1$ in (19), we have the following frozen three-step Steffensen method:

$$
\phi_{\mathcal{B}(\beta=\alpha=1)}:\left\{\begin{aligned}
y & =x-\theta^{-1} f(x) \\
z & =y-\theta^{-1} f(y) \\
X & =z-\theta^{-1} f(z)
\end{aligned}\right.
$$

In this case, the efficiency index is $E I_{\mathcal{B}(\beta=\alpha=1)}=3^{1 / 4}=1.316$, whereas $E I_{\mathcal{B}(\beta=1 / \alpha)}=4^{1 / 4}=1.414$.

\subsection{Third family}

The last family of iterative methods of highest order for $\theta_{1}$ and $\theta_{2}$ is:

$$
\phi_{\mathcal{C}}:\left\{\begin{aligned}
y & =x-\theta^{-1} f(x), \\
z & =y-\theta^{-1}(-f(x)+\alpha f(y)), \\
X & =z-\theta^{-1}((1-\beta) f(x)+(1-\alpha+\alpha \beta) f(y)+\beta f(z)) .
\end{aligned}\right.
$$

The three-step iterative method $\phi_{\mathcal{C}}$ given in (22) has the following error equation:

$$
\left|e_{n+1}\right|= \begin{cases}A_{2}^{2}|(2 e-\widetilde{e}-\alpha \beta \widetilde{e})(e+\widetilde{e}) e|+O_{4}(e, \widetilde{e}) & \text { for } \theta=\theta_{1}, \\ A_{2}^{2}|(2 e+\widetilde{e}+\alpha \beta \widetilde{e})(e-\widetilde{e}) e|+O_{4}(e, \widetilde{e}) & \text { for } \theta=\theta_{2} .\end{cases}
$$

In both cases, the local order of convergence is at least three and the efficiency index is $E I_{\mathcal{C}}=3^{1 / 4}=1.316$.

\subsection{Fourth family}

In a similar way as the beginning of this section, developing again the error of iteration function $\phi$ with the divided difference $\theta_{3}, e_{n+1}=X-x^{*}$, in powers of $e_{n}=e=x-x^{*}$ until fifth order and cancelling the coefficients of the terms up to third order, we have:

- coefficients of $e$ :

$$
\alpha_{1}+\beta_{1}-\alpha_{1} \beta_{3}=0,
$$

- coefficients of $A_{2} e^{2}$ :

$$
-1-\alpha_{1}+\alpha_{2}-\beta_{1}+\beta_{2}+\beta_{3}+\left(3 \alpha_{1}+\alpha_{1}^{2}-\alpha_{2}\right) \beta_{3}=0,
$$

- coefficients of $A_{3} e \widetilde{e}^{2}$ :

$$
-1-\alpha_{1}+\alpha_{2}-\beta_{1}+\beta_{2}+\beta_{3}+\left(2 \alpha_{1}-\alpha_{2}\right) \beta_{3}=0,
$$

- coefficients of $A_{2}^{2} e^{3}$ :

$$
-1-\alpha_{1}+2 \alpha_{2}-\beta_{1}+2 \beta_{2}+2 \beta_{3}+\left(5 \alpha_{1}-3 \alpha_{2}+2 \alpha_{1}^{2}-\alpha_{1} \alpha_{2}\right) \beta_{3}=0,
$$

- coefficients of $A_{3} e^{3}$ :

$$
2+2 \alpha_{1}-2 \alpha_{2}+2 \beta_{1}-2 \beta_{2}-2 \beta_{3}+\left(\alpha_{1}^{3}-5 \alpha_{1}+2 \alpha_{2}\right) \beta_{3}=0 .
$$

In addition, we get one set of solutions, called $\mathcal{D}$, that has fourth order and is defined by

$$
\mathcal{D}=\left\{\alpha_{1}=0, \alpha_{2}=\alpha, \beta_{1}=0, \beta_{2}=-\frac{(1-\alpha)^{2}}{\alpha}, \beta_{3}=\frac{1}{\alpha}\right\} .
$$

The new family of at least fourth order of convergence, $\phi_{\mathcal{D}}$, is the same as $\phi_{\mathcal{B}}$ with $\beta=1 / \alpha$ (family (19)), but with $\theta=\theta_{3}$. That is,

$$
\phi_{\mathcal{D}}:\left\{\begin{aligned}
y & =x-\theta^{-1} f(x), \\
z & =y-\alpha \theta^{-1} f(y), \\
X & =z-\frac{1}{\alpha} \theta^{-1}\left(-(1-\alpha)^{2} f(y)+f(z)\right),
\end{aligned}\right.
$$

whose error equation is

$$
\left|e_{n+1}\right|=|\alpha-5|\left|A_{2}^{3}\right| e_{n}^{4}+O_{5}(e)
$$

and the efficiency index $E I_{\mathcal{D}}=4^{1 / 5}=1.320$. Setting $\alpha=1$, we find again the classical frozen three-step Steffensen method, but now with $\theta=\theta_{3}$. 
In particular, taking $\alpha=5$ in (23), we have the following three-step frozen Steffensen method of fifth order:

$$
\phi_{\mathcal{D}(\alpha=5)}:\left\{\begin{aligned}
y & =x-\theta^{-1} f(x), \\
z & =y-5 \theta^{-1} f(y), \\
X & =z-\frac{1}{5} \theta^{-1}(f(z)-16 f(y)),
\end{aligned}\right.
$$

whose error equation is $\left|e_{n+1}\right|=2\left|A_{2}^{2}\right|\left|7 A_{2}^{2}+2 A_{3}\right|\left|e_{n}\right|^{5}+O_{6}(e)$ and the efficiency index $E I_{\mathcal{D}}=5^{1 / 5}=1.380$.

Eventually, we recall that we are interested in a generalization of the results of this section to the multidimensional case. We choose the iterative methods with the highest convergence order and minimum computational cost:

Setting by $\phi_{1}^{(j)}, j=1,2$, that is the Steffensen method with divided differences $\theta_{j}, j=1,2$, respectively, we have $E I_{1}=2^{1 / 3}$.

Choosing as $\phi_{2}^{(j)}, j=1,2$, the family $\phi_{\mathcal{A}}$, we obtain $E I_{2}=3^{1 / 3}$.

Denoting by $\phi_{3}^{(j)}, j=1,2$, the iteration family $\phi_{\mathcal{B}}(\beta=1 / \alpha)$, we obtain $E I_{3}=4^{1 / 3}$.

Taking $\phi_{4}^{(3)}=\phi_{\mathcal{D}}$, where the divided difference $\theta_{3}$ is used, we get $E I_{4}=4^{1 / 5}$.

Henceforth, in the multidimensional case, we consider the following iterative families:

$$
\begin{aligned}
& \Phi_{1}:\left\{X=x-\Theta^{-1} F(x), \quad \Phi_{2}:\left\{\begin{array}{l}
y=x-\Theta^{-1} F(x), \\
X=y-\Theta^{-1} F(y),
\end{array} \quad \Phi_{3}:\left\{\begin{array}{l}
y=x-\Theta^{-1} F(x), \\
z=y-\Theta^{-1} F(y), \\
X=z-\Theta^{-1} F(z),
\end{array}\right.\right.\right. \\
& \Phi_{4}:\left\{\begin{array}{l}
y=x-\Theta^{-1} F(x), \\
z=y-\alpha \Theta^{-1} F(y), \\
X=z-\frac{1}{\alpha} \Theta^{-1}\left(F(z)-(1-\alpha)^{2} F(y)\right)=\frac{1}{\alpha^{2}}\left((2 \alpha-1) z+(\alpha-1)^{2} y\right)-\frac{1}{\alpha} \Theta^{-1} F(z) .
\end{array}\right.
\end{aligned}
$$

Iterative method $\Phi_{1}$ corresponds to Steffensen method and is the first step of (2).

Algorithm $\Phi_{2}$, called frozen two-step Steffensen method, is exactly $\phi_{\mathcal{A}}$ with $\alpha=0$ and $\beta=1$ (see (17)) in the multidimensional case.

Algorithm $\Phi_{3}$, called frozen three-step Steffensen method, is a generalization of $\phi_{\mathcal{B}}$ with $\beta=1 / \alpha=1$ (see $(21)$ ).

The last algorithm, $\Phi_{4}$, can be deduced from $\phi_{\mathcal{B}}$ with $\beta=1 / \alpha$ (see (19)) when operators $\Theta_{1}$ or $\Theta_{2}$ are used, or from $\phi_{\mathcal{D}}$ when operator $\Theta_{3}$ is used (see (23)).

\section{Maximum local order of convergence in the multidimensional case}

We consider the one-parametric iteration family $\Phi_{4}$ and we firstly analyze the local order of convergence. Note that the algorithms $\Phi_{\ell}, 1 \leq \ell \leq 3$, are particular cases of family $\Phi_{4}$ with $\alpha=1$ and/or less steps.

\subsection{Operators $\Theta_{1}$ and $\Theta_{2}$}

For operator $\Theta_{1}$, we have the expression of the error of the first step of $\Phi_{4}$ in (12); namely, $e_{y}^{+}=y^{+}-x^{*}$. Moreover, we have the error equation for $\Phi_{1}$ using operator $\Theta_{1}$, since $y^{+}=\Phi_{1}^{+}(x)$. In a similar way, from (13), we obtain the same results for $e_{y}^{-}=y^{-}-x^{*}$ and $y^{-}=\Phi_{1}^{-}(x)$.

Subtracting $x^{*}$ from both sides of the second step of $\Phi_{4}$, iteration (25), taking the inverse operators (9)-(10) and considering $F(y)=\Gamma\left(e_{y}+A_{2} e_{y}^{2}+O_{3}\left(e_{y}\right)\right)$, we have the following respective errors: 


$$
\begin{aligned}
e_{z}^{+}=e_{y}^{+}-\alpha \Theta_{1}^{-1} F\left(y^{+}\right)= & (1-\alpha) e_{y}^{+}+\alpha A_{2}(2 e+\widetilde{e}) e_{y}^{+}-\alpha A_{2}\left(e_{y}^{+}\right)^{2}+\alpha A_{3}\left(3 e^{2}+3 e \widetilde{e}+\widetilde{e}^{2}\right) e_{y}^{+} \\
& +\alpha A_{2} \Gamma A_{2} e^{2} e_{y}^{+}-\alpha\left\{A_{2}(2 e+\widetilde{e})\right\}^{2} e_{y}^{+}+O_{5}(e, \widetilde{e}), \\
e_{z}^{-}=e_{y}^{-}-\alpha \Theta_{2}^{-1} F\left(y^{-}\right)= & (1-\alpha) e_{y}^{-}+\alpha A_{2}(2 e-\widetilde{e}) e_{y}^{+}-\alpha A_{2}\left(e_{y}^{-}\right)^{2}+\alpha A_{3}\left(3 e^{2}-3 e \widetilde{e}+\widetilde{e}^{2}\right) e_{y}^{+} \\
& -\alpha A_{2} \Gamma A_{2} e^{2} e_{y}^{+}-\alpha\left\{A_{2}(2 e+\widetilde{e})\right\}^{2} e_{y}^{+}+O_{5}(e, \widetilde{e}) .
\end{aligned}
$$

Note that, from (26) with $\alpha=1$, we have the error expression for $\Phi_{2}$ using operator $\Theta_{1}$, since $z^{+}=\Phi_{2}^{+}(x)$. In a similar way, from (27) we obtain the same results for $e_{z}^{-}=z^{-}-x^{*}$ and $z^{-}=\Phi_{2}^{-}(x)$.

Recall that the third step of $\Phi_{4}, X=z-\frac{1}{\alpha} \Theta^{-1}\left(F(z)-(1-\alpha)^{2} F(y)\right)$, is equivalent to

$$
X=\frac{1}{\alpha^{2}}\left((2 \alpha-1) z+(\alpha-1)^{2} y\right)-\frac{1}{\alpha} \Theta^{-1} F(z)
$$

where $\Theta^{-1} F(y)$ is replaced in function of $y$ and $z$ from the second step of (25). Subtracting again $x^{*}$ from both sides of the previous equation (28), taking the inverse operators given in (9)-(10) and expanding $F(z)=\Gamma\left(e_{z}+A_{2} e_{z}^{2}+O_{3}\left(e_{z}\right)\right)$, we have the following respective errors

$$
\begin{aligned}
& X^{+}-x^{*}=e_{X}^{+}=e_{z}^{+}-\alpha \Theta_{1}^{-1} F\left(z^{+}\right)=(1-\alpha) A_{2}\left(e_{y}^{+}\right)^{2}+\left\{A_{2}(2 e+\widetilde{e})\right\}^{2} e_{y}^{+}+O_{5}(e, \widetilde{e}), \\
& X^{-}-x^{*}=e_{X}^{-}=e_{z}^{-}-\alpha \Theta_{2}^{-1} F\left(z^{-}\right)=(1-\alpha) A_{2}\left(e_{y}^{-}\right)^{2}+\left\{A_{2}(2 e-\widetilde{e})\right\}^{2} e_{y}^{+}+O_{5}(e, \widetilde{e}),
\end{aligned}
$$

where we recall, see respectively (12) and (13), that

$$
\begin{aligned}
& e_{y}^{+}=A_{2}(e+\widetilde{e}) e+O_{3}(e, \widetilde{e}), \\
& e_{y}^{-}=A_{2}(e-\widetilde{e}) e+O_{3}(e, \widetilde{e}) .
\end{aligned}
$$

The error equation for $\Phi_{4}^{+}$is given by (29), where $e_{n+1}=e_{X}^{+}=\Phi_{4}^{+}\left(x_{n}\right)-x^{*}$ and $e=x-x^{*}$. Analogously, the error of $\Phi_{4}^{-}\left(x_{n}\right)$ is given by (30), where $e_{n+1}=e_{X}^{-}=\Phi_{4}^{-}\left(x_{n}\right)-x^{*}$. The local order of convergence of $\Phi_{4}$ is at least four in both cases and the error expressions (29)-(32) are the same as in the scalar case (see (20)).

\subsection{Operator $\Theta_{3}$}

For operator $\Theta_{3}$ we have the expression of the error of the first step of method $\Phi_{4}$ in (14). Say, $e_{y}^{\mp}=y^{\mp}-x^{*}$. Moreover, we have the equation of the error for $\Phi_{1}$ using operator $\Theta_{3}$, since $y^{\mp}=\Phi_{1}^{\mp}(x)$.

Subtracting $x^{*}$ from both sides of the second step of $\Phi_{4}$, taking the inverse operator (11) and considering $F(y)=\Gamma\left(e_{y}+A_{2} e_{y}^{2}+O_{3}\left(e_{y}\right)\right)$, we have the following error equation:

$$
\begin{aligned}
e_{z}^{\mp}=e_{y}^{\mp}-\alpha \Theta_{3}^{-1} F\left(y^{\mp}\right)= & (1-\alpha) e_{y}^{\mp}+2 \alpha A_{2} e e_{y}^{\mp}-\alpha A_{2}\left(e_{y}^{+\mp}\right)^{2}+\alpha A_{3}\left(3 e^{2}+\widetilde{e}^{2}\right) e_{y}^{\mp} \\
& -4 \alpha\left\{A_{2} e\right\}^{2} e_{y}^{+}+O_{5}(e, \widetilde{e}) .
\end{aligned}
$$

Note that, from (33) with $\alpha=1$, we have the error expression for $\Phi_{2}$ using operator $\Theta_{3}$, since $z^{\mp}=\Phi_{2}^{\mp}(x)$.

Subtracting again $x^{*}$ from both sides of the third step of $\Phi_{4}$, taking the inverse operators given in (11) and expanding $F(z)=\Gamma\left(e_{z}+A_{2} e_{z}^{2}+O_{3}\left(e_{z}\right)\right)$, we obtain

$$
X^{\mp}-x^{*}=e_{X}^{\mp}=e_{z}^{\mp}-\alpha \Theta_{3}^{-1} F\left(z^{\mp}\right)=(1-\alpha) A_{2}\left(e_{y}^{\mp}\right)^{2}+4\left\{A_{2} e\right\}^{2} e_{y}^{\mp}+O_{5}(e, \widetilde{e}) .
$$

From (14), we take $e_{y}^{\mp}=A_{2} e^{2}+O_{3}(e, \widetilde{e})$ and substituting in (34), we get

$$
e_{n+1}=e_{X}^{\mp}=\Phi_{4}^{\mp}\left(x_{n}\right)-x^{*}=(1-\alpha) A_{2}\left(A_{2} e^{2}\right)^{2}+4\left\{A_{2} e\right\}^{2} A_{2} e^{2}+O_{5}(e, \widetilde{e}) .
$$


The error equation for $\Phi_{4}^{\mp}$ is given by (35) and the terms of fourth order are canceled if and only if equation (35) is expressed in the scalar case and $\alpha=5$. It is worth seeing with more detail. In a notation more classical, we write the fourth-order terms of the right side of (35) in the following way:

$$
(1-\alpha) A_{2}\left(A_{2} e^{2}\right)\left(A_{2} e^{2}\right)+4\left\{A_{2} e\right\}^{2} A_{2} e^{2}=(1-\alpha) A_{2}\left(A_{2}(e, e), A_{2}(e, e)\right)+4 A_{2}\left(e, A_{2}\left(e, A_{2}(e, e)\right)\right) .
$$

Error equation (24) is not true except for the scalar case. Hence, the local order of convergence of $\Phi_{4}$, using operator $\Theta_{3}$, is, independently of $\alpha$, at least four and error expression (35) is very different from the scalar case.

Now, from the results found in the two last sections, we can establish the following result.

Theorem 3.1 Let $F: D \subseteq \mathbb{R}^{m} \longrightarrow \mathbb{R}^{m}$ be a nonlinear function defined on a nonempty open convex domain $D$. Assume that $F$ has, at least, third-order Fréchet derivatives with continuity on $D$. We suppose that there exists $[u, v ; F] \in \mathcal{L}(D, D)$, for all $u, v \in D(u \neq v)$. The iterative families $\Phi_{k}, 1 \leq k \leq 4$, where $\Theta$ represents the operators $[x, x+F(x) ; F],[x-F(x), x ; F]$ or $[x-F(x), x+F(x) ; F]$, for $x \in D$, have order of convergence at least two for $\Phi_{1}$, with the error equations given in (12)-(14), at least three for $\Phi_{2}$, with the error equations given in (26), (27) and (33), and at least four for $\Phi_{3}$ and $\Phi_{4}$, with the error equations given in (29)-(32) and (35).

Note that, from the independence of $\alpha$, we take $\alpha=1$ and compare the efficiency of the methods $\Phi_{1}, \Phi_{2}$ and $\Phi_{3}=\Phi_{4}$. In particular, for $\Phi_{3}$, we have

$$
\begin{aligned}
& X^{+}-x^{*}=\left\{A_{2}(2 e+\widetilde{e})\right\}^{2} A_{2}(e+\widetilde{e}) e+O_{5}(e, \widetilde{e}), \\
& X^{-}-x^{*}=\left\{A_{2}(2 e-\widetilde{e})\right\}^{2} A_{2}(e-\widetilde{e}) e+O_{5}(e, \widetilde{e}), \\
& X^{\mp}-x^{*}=4\left\{A_{2} e\right\}^{2} A_{2} e^{2}+O_{5}(e, \widetilde{e}) .
\end{aligned}
$$

\section{Optimal computational efficiency}

It is well-known that the efficiency of an iterative method cannot only be measured from the operational cost. It is clear that the number of evaluations of functions that are needed to apply the algorithm, that defines the iterative method, plays an important role when it comes to measuring the efficiency of the method. In the scalar case, Traub defines in [18] the index $\rho^{1 / \vartheta}$, where $\rho$ is the local order of convergence of the method and $\vartheta$ represents the number of the evaluations of functions necessary to apply the method. Then, it seems clear that a more real measurement of the efficiency of an iterative method is through the conjunction of these two quantities. So, to compare the efficiencies of the iterative methods, we can use the following computational efficiency index $(C E I)$ :

$$
C E I(\mu, m, \ell)=\rho^{\frac{1}{\mathcal{C}(\mu, m, \ell)}},
$$

where $\rho$ is the $R$-order of convergence and $\mathcal{C}(\mu, m, \ell)$ is the computational cost of an iterative method, which is given by

$$
\mathcal{C}(\mu, m, \ell)=a(m) \mu+p(m, \ell)
$$

where $\mu$ is the ratio between products and evaluations of scalar functions of $F(x)$, respectively, that are required to express $\mathcal{C}_{i}(\mu, m, \ell)$ in terms of products. Parameter $\ell$ is the ratio between products and quotients, $a(m)$ is the number of evaluations of $F(x)$ and $p(m, \ell)$ is the number of products needed in the operations of the iterative method per iteration. Notice that the definition of $C E I$ given in (36) is a generalization of the scalar case to several variables (see $[9,10,14]$ ).

In this work, we use the following numerical definition of divided difference operator [14]:

$$
[\mathbf{u}, \mathbf{v} ; F]_{i j}=\frac{1}{u_{j}-v_{j}}\left(F_{i}\left(u_{1}, \ldots, u_{j}, v_{j+1}, \ldots, v_{m}\right)-F_{i}\left(u_{1}, \ldots, u_{j-1}, v_{j}, \ldots, v_{m}\right)\right),
$$


where $\mathbf{u}=\left(u_{1}, u_{2}, \ldots, u_{m}\right)^{T}$ and $\mathbf{v}=\left(v_{1}, v_{2}, \ldots, v_{m}\right)^{T}$, for $\mathbf{u}, \mathbf{v} \in \mathbb{R}^{m},[\mathbf{u}, \mathbf{v} ; F]=\left([\mathbf{u}, \mathbf{v} ; F]_{i j}\right)_{i, j=1}^{m} \in$ $\mathcal{L}\left(\mathbb{R}^{m}, \mathbb{R}^{m}\right)$.

Taking into account that the computational cost of $\Theta_{3}$ is higher than those of $\Theta_{1}$ and $\Theta_{2}$ and the orders of iterative methods $\Phi_{k}^{+}, \Phi_{k}^{-}$and $\Phi_{k}^{\mp}, 1 \leq k \leq 3$, are the same, we then reduce the study to $\Phi^{+}$, that uses operator $\Theta_{1}$ and is denoted by $\Phi$. Note that, for iterative method $\Phi^{-}$, we obtain the same results.

From the previous results, we have, for $1 \leq k \leq 3$,

$$
\mathcal{C}_{\Phi_{k}}(\mu, m, \ell)=m(m+k) \mu+\frac{m}{6}\left(2 m^{2}-3 m+1\right)+m(m-1) k+\frac{m}{2}(3 m-1) \ell+m k \ell, \quad \rho_{\Phi_{k}}=k+1,
$$

where $a(m)=m(m+k)$, since we take $m^{2}$ evaluations of component functions of $F$ to compute operator (37) and $m k$ when we evaluate $k$ times the function $F$. In the unique $L U$ factorization that we use, we have $\frac{m}{6}\left(2 m^{2}-3 m+1\right)$ products and $\frac{m}{2}(m-1)$ quotients. Moreover, we take into account $m^{2}$ quotients in the computation of $(37)$ and $m(m-1) k$ products and $m k$ quotients in the $k$ resolutions of two triangular linear systems.

In order to compare iterative methods $\Phi_{k}, 1 \leq k \leq 3$, we define the following ratio

$$
R_{i, j}=\frac{\log C E I_{\Phi_{i}}(\mu, m, \ell)}{\log C E I_{\Phi_{j}}(\mu, m, \ell)}=\frac{\log \rho_{\Phi_{i}}}{\log \rho_{\Phi_{j}}} \frac{\mathcal{C}_{\Phi_{j}}(\mu, m, \ell)}{\mathcal{C}_{\Phi_{i}}(\mu, m, \ell)}
$$

\section{1 $\Phi_{1}$ versus $\Phi_{2}$}

To compare iterative method $\Phi_{1}$ with method $\Phi_{2}$, we consider the following ratio:

$$
R_{1,2}=\frac{\log 2}{\log 3} \frac{2 m^{2}+9 m+9 \ell m+6 m \mu+12 \mu-11+9 \ell}{2 m^{2}+3 m+9 \ell m+6 m \mu+6 \mu-5+3 \ell},
$$

where $R_{1,2}<1$ for all $\ell \geq 1$ and $m \geq 2$ (see figure 1 ). Notice that, for $\ell \geq 1$, the curve $R_{1,2}=1$ has a zero $m_{0}$ with $m_{0}<1$.

\section{2 $\Phi_{2}$ versus $\Phi_{3}$}

To compare iterative method $\Phi_{2}$ with method $\Phi_{3}$, we consider the following ratio:

$$
R_{2,3}=\frac{\log 3}{\log 4} \frac{2 m^{2}+15 m+9 \ell m+6 m \mu+18 \mu-17+15 \ell}{2 m^{2}+9 m+9 \ell m+6 m \mu+12 \mu-11+9 \ell},
$$

where $R_{2,3}<1$, for all $m \geq 3$ and $\ell \geq 1$, or $m=2$ and $\mu>\mu_{0}>0$ (see figure 2 ). Notice that

$$
\mu_{0}=\frac{1}{2} \frac{7 \log 3-10 \log 2+(11 \log 3-18 \log 2) \ell}{-5 \log 3+8 \log 2} \approx 7.28005-3.76002 \ell,
$$

and $\mu_{0}>0$ if $\ell<\ell_{0} \approx 1.93617$.

\section{3 $\Phi_{1}$ versus $\Phi_{3}$}

From the two preceding comparisons we can deduce that $R_{1,3}<1$ for all $m \geq 3$ and $\ell \geq 1$.

For $m=2$, the curve $R_{1,3}=1$ is the line $2 \mu=1-3 \ell$. As $\ell \geq 1$, we have also $R_{1,3}<1$.

Summarizing these previous results we can establish the following result.

Theorem 4.1 The CEI of methods $\Phi_{1}, \Phi_{2}$ and $\Phi_{3}$ satisfy the following:

(a) For all $m \geq 3$ and $\ell \geq 1$, we have $C E I_{3}>C E I_{2}>C E I_{1}$.

(b) For $m=2$, we have

- $C E I_{3}>C E I_{2}>C E I_{1}$ if $\mu>\mu_{0}>0$,

- $C E I_{2}>C E I_{3}>C E I_{1}$ if $\mu \in\left(0, \mu_{0}\right)$. 


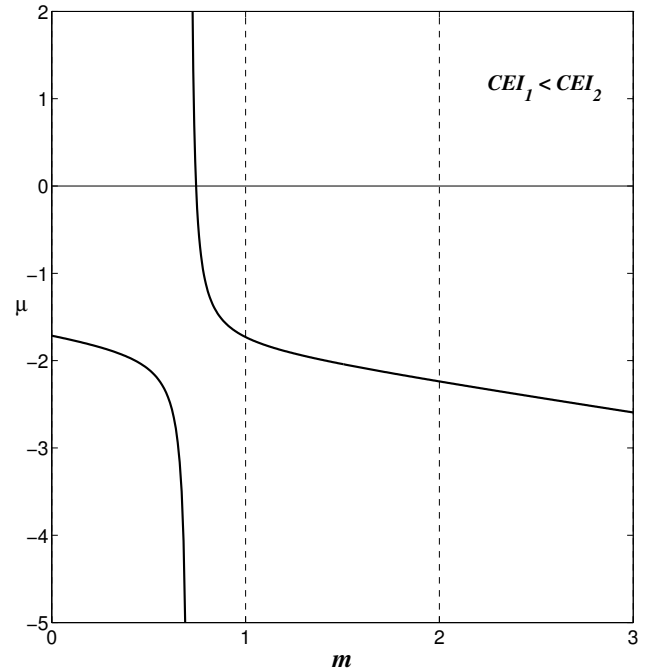

Figure 1: The boundary $R_{1,2}=1$ for $0 \leq m \leq 3$ and $\ell=1.731$.

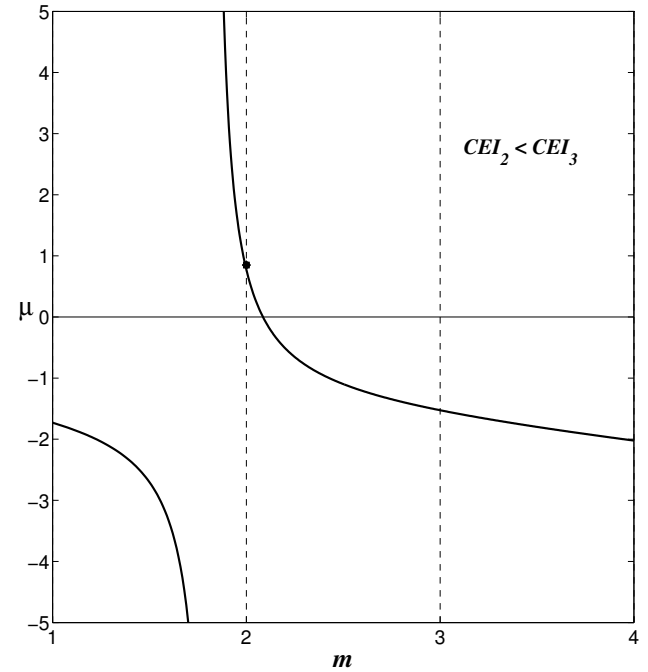

Figure 2: The boundary $R_{2,3}=1$ for $1 \leq m \leq 4$ and $\ell=1.731$.

\section{Semilocal convergence of the optimal iterative methods}

In this section, we study the semilocal convergence of the optimal iterative methods considered in the previous study. First, as it is known (see [14]), in $\mathbb{R}^{m}$ we can consider that there exists a divided difference of first order, $[x, y ; F]$, for each pair of distinct points $x, y \in \mathbb{R}^{m}$. So, for the sequence $\left\{x_{n}\right\}$ given by (46), it is clear that every divided difference of first order $\left[x_{k}, x_{k}+F\left(x_{k}\right) ; F\right]$ exists, except for $x_{k}=x_{k}+F\left(x_{k}\right)$, since in such case it is evident that $x_{k}$ is a solution of $F(x)=0$, so that $x_{n}=x_{k}$, for all $n \geq k$, and then the sequence $\left\{x_{n}\right\}$ converges to $x_{k} \equiv x^{*}$, which is a solution of $F(x)=0$. For the semilocal convergence study, we consider the usually conditions. So, let $F: D \subseteq \mathbb{R}^{m} \longrightarrow \mathbb{R}^{m}$ be a nonlinear function defined on a nonempty open convex domain $D$. Given $x_{0}, x_{0}+F\left(x_{0}\right) \in D$, we observe that $x_{0} \neq x_{0}+F\left(x_{0}\right)$, since $x_{0}$ is a solution of $F(x)=0$ in other case, and consequently, $x_{n}=x_{0}$ for all $n \in \mathbb{N}$. After that, we suppose the following conditions:

(C1) $\left\|F\left(x_{0}\right)\right\| \leq \delta$,

(C2) $A_{0}^{-1}=\left[x_{0}, x_{0}+F\left(x_{0}\right) ; F\right]^{-1}$ exists and is such that $\left\|A_{0}^{-1}\right\| \leq \beta$,

(C3) $\|[x, y ; F]-[u, v ; F]\| \leq K(\|x-u\|+\|y-v\|) ; K \geq 0 ; x, y, u, v \in D ; x \neq y ; u \neq v$.

First, we consider Steffensen's method:

$$
\left\{\begin{array}{l}
x_{0} \in D, \\
x_{n+1}=x_{n}-\left[x_{n}, x_{n}+F\left(x_{n}\right) ; F\right]^{-1} F\left(x_{n}\right), \quad n \geq 0 .
\end{array}\right.
$$

From now on, we denote $A_{n}=\left[x_{n}, x_{n}+F\left(x_{n}\right) ; F\right]$.

Next, we give the semilocal convergence result given in [7] for Steffensen's method.

Theorem 5.1 Let $F: D \subseteq \mathbb{R}^{m} \longrightarrow \mathbb{R}^{m}$ be a nonlinear function defined on a nonempty open convex domain D. Suppose that conditions (C1)-(C3) are satisfied. If the equation

$$
t=\frac{(1-g(t)) \eta}{1-(M+g(t))}+M \delta
$$

where $M=K \beta \delta(1+\beta), g(t)=K \beta(2 t+\delta)$ and $\eta=\beta \delta$, has at least one positive real root and the smallest positive real root, denoted by $R$, satisfies

$$
M+g(R)<1
$$


and $B\left(x_{0}, R\right) \subset D$, then Steffensen's method (39), starting at $x_{0}$, is well-defined and converges to a solution $x^{*}$ de $F(x)=0$. Moreover, the solution $x^{*}$ and the iterates $x_{n}$ belong to $\overline{B\left(x_{0}, R\right)}$ and $x^{*}$ is unique in $\overline{B\left(x_{0}, R\right)}$.

We continue with the analyse the semilocal convergence of the iterative method called frozen two-step Steffensen method, whose algorithm is:

$$
\left\{\begin{array}{l}
x_{0} \in D, \\
y_{n}=x_{n}-\left[x_{n}, x_{n}+F\left(x_{n}\right) ; F\right]^{-1} F\left(x_{n}\right), \\
x_{n+1}=y_{n}-\left[x_{n}, x_{n}+F\left(x_{n}\right) ; F\right]^{-1} F\left(y_{n}\right), \quad n \geq 0 .
\end{array}\right.
$$

We began with a technical lemma that is used later and whose proof is immediate from algorithm (42) and the definition of the divided difference of first order $[v, w ; F], v, w \in D$, which is a bounded linear operator such that (see [14])

$$
[v, w ; F]: D \subseteq \mathbb{R}^{m} \longrightarrow \mathbb{R}^{m} \quad \text { and } \quad[v, w ; F](v-w)=F(v)-F(w) .
$$

Lemma 5.1 Let $\left\{x_{n}\right\}$ be the sequence given by the frozen two-step Steffensen method given in (42). If $x_{n} \neq$ $y_{n-1}, y_{n}$ with $x_{n}, y_{n} \in D$, then

$$
\begin{aligned}
& F\left(y_{n}\right)=\left(\left[y_{n}, x_{n} ; F\right]-A_{n}\right)\left(y_{n}-x_{n}\right), \\
& F\left(x_{n}\right)=\left(\left[x_{n}, y_{n-1} ; F\right]-A_{n-1}\right)\left(x_{n}-y_{n-1}\right) .
\end{aligned}
$$

Next, we present the new semilocal convergence result for method (42) under conditions $(C 1)-(C 3)$.

Theorem 5.2 Let $F: D \subseteq \mathbb{R}^{m} \longrightarrow \mathbb{R}^{m}$ be a nonlinear function defined on a nonempty open convex domain D. Suppose that conditions (C1)-(C3) are satisfied. If the equation

$$
t=\left(1+\frac{M(1-g(t))}{1-(g(t)+h(t))}\right) \eta+M h(t) \delta=0,
$$

where $M=K \beta \delta(1+\beta), g(t)=K \beta(2 t+\delta), h(t)=K \beta(t+\eta+\delta)$ and $\eta=\beta \delta$, has at least one positive real root and the smallest positive real root, denoted by $R$, satisfies

$$
g(R)+h(R)<1
$$

and $B\left(x_{0}, R\right) \subset D$, then the frozen two-step Steffensen method given in (42), starting at $x_{0}$, is well-defined and converges to a solution $x^{*}$ of $F(x)=0$. Moreover, the solution $x^{*}$ and the iterates $x_{n}$ belong to $\overline{B\left(x_{0}, R\right)}$ and $x^{*}$ is unique in $\overline{B\left(x_{0}, R\right)}$.

Proof. We begin by proving that the sequence given by method (42) is well-defined and $x_{n} \in B\left(x_{0}, R\right) \subset D$ for all $n \in \mathbb{N}$. Note that the smallest positive real root $R$ of equation (43) satisfies

$$
R=(1+P) \eta+M h(R) \delta,
$$

where $P=\frac{M(1-g(R))}{1-(g(R)+h(R))} \in(0,1)$, as we can see easily from (44).

From (C1)-(C3), it follows that $y_{0}$ and $x_{1}$ are well-defined. Besides, from (C3) and taking into account $F\left(y_{0}\right)=\left(\left[y_{0}, x_{0} ; F\right]-A_{0}\right)\left(y_{0}-x_{0}\right)$, by Lemma 5.1 , we obtain

$$
\left.\left\|F\left(y_{0}\right)\right\| \leq K\left(\left\|y_{0}-x_{0}\right\|+\left\|F\left(x_{0}\right)\right\|\right)\right)\left\|y_{0}-x_{0}\right\|<M \delta<\delta,
$$

since $M<1,\left\|x_{1}-y_{0}\right\|<M \eta$ and $\left\|x_{1}-x_{0}\right\|<(1+M) \eta<R$, as a consequence of (45). Thus, $x_{1} \in B\left(x_{0}, R\right)$. In addition, by Lemma 5.1 , it follows $F\left(x_{1}\right)=\left(\left[x_{1}, y_{0} ; F\right]-A_{0}\right)\left(x_{1}-y_{0}\right)$, so that

$$
\left\|F\left(x_{1}\right)\right\| \leq K\left(\left\|x_{1}-x_{0}\right\|+\left\|y_{0}-x_{0}\right\|+\left\|F\left(x_{0}\right)\right\|\right)\left\|x_{1}-y_{0}\right\|<M h(R) \delta<\delta,
$$


since $M<h(R)<1$, so that, again by (45), we have

$$
\left\|x_{1}+F\left(x_{1}\right)-x_{0}\right\| \leq\left\|x_{1}-x_{0}\right\|+\left\|F\left(x_{1}\right)\right\|<(1+M) \eta+M h(R) \delta<R
$$

and $x_{1}+F\left(x_{1}\right) \in B\left(x_{0}, R\right)$. Next, by (C3), we obtain

$\left\|I-A_{0}^{-1} A_{1}\right\| \leq\left\|A_{0}^{-1}\right\|\left\|A_{0}-A_{1}\right\| \leq \beta K\left(\left\|x_{1}+F\left(x_{1}\right)-x_{0}\right\|+\left\|x_{1}-x_{0}\right\|+\left\|F\left(x_{0}\right)\right\|\right) \leq \beta K(2 R+\delta)=g(R)<1$,

and, by the Banach lemma on invertible operators, there exists $A_{1}^{-1}$ and is such that

$$
\left\|A_{1}^{-1}\right\| \leq \frac{\beta}{1-g(R)} .
$$

After that, from $M<h(R)<g(R)<1$ and (44), we see that

$$
\begin{gathered}
\left\|y_{1}-x_{1}\right\| \leq\left\|A_{1}^{-1}\right\|\left\|F\left(x_{1}\right)\right\| \leq M\left(\frac{h(R)}{1-g(R)}\right) \eta<\eta, \\
\left\|y_{1}-x_{0}\right\| \leq\left\|y_{1}-x_{1}\right\|+\left\|x_{1}-x_{0}\right\|<(1+P) \eta<R,
\end{gathered}
$$

so that $y_{1} \in B\left(x_{0}, R\right)$. In addition, again from $M<h(R)<g(R)<1$ and (44), it follows

$$
\begin{gathered}
\left\|F\left(y_{1}\right)\right\| \leq K\left(\left\|y_{1}-x_{1}\right\|+\left\|F\left(x_{1}\right)\right\|\right)\left\|y_{1}-x_{1}\right\|<M^{2}\left(\frac{h(R)}{1-g(R)}\right) \delta<\delta, \\
\left\|x_{2}-y_{1}\right\| \leq\left\|A_{1}^{-1}\right\|\left\|F\left(y_{1}\right)\right\|<M\left(\frac{h(R)}{1-g(R)}\right)^{2} \eta<\eta, \\
\left\|x_{2}-x_{1}\right\| \leq\left\|x_{2}-y_{1}\right\|+\left\|y_{1}-x_{1}\right\|<P \eta<R, \\
\left\|x_{2}-x_{0}\right\| \leq\left\|x_{2}-y_{1}\right\|+\left\|y_{1}-x_{0}\right\|<(1+P) \eta<R .
\end{gathered}
$$

Therefore, $x_{2} \in B\left(x_{0}, R\right)$. Moreover, from (44), we have

$$
\left\|F\left(x_{2}\right)\right\| \leq K\left(\left\|x_{2}-x_{1}\right\|+\left\|y_{1}-x_{1}\right\|+\left\|F\left(x_{1}\right)\right\|\right)\left\|x_{2}-y_{1}\right\|<M^{2}\left(\frac{h(R)}{1-g(R)}\right)^{2} \delta<\delta,
$$

so that

$$
\left\|x_{2}+F\left(x_{2}\right)-x_{0}\right\| \leq\left\|x_{2}-x_{0}\right\|+\left\|F\left(x_{2}\right)\right\|<(1+P) \eta+M h(R) \delta=R
$$

and $x_{2}+F\left(x_{2}\right) \in B\left(x_{0}, R\right)$.

Now, we assume the following:

$$
\begin{aligned}
& \cdot\left\|A_{n}^{-1}\right\| \leq \frac{\beta}{1-g(R)}, \\
& \cdot\left\|y_{n}-x_{n}\right\|<M\left(\frac{h(R)}{1-g(R)}\right)^{2 n-1} \eta, \\
& \cdot\left\|F\left(y_{n}\right)\right\|<K(\eta+\delta)\left\|y_{n}-x_{n}\right\|, \\
& \cdot\left\|x_{n+1}-y_{n}\right\|<M\left(\frac{h(R)}{1-g(R)}\right)^{2 n} \eta, \\
& \cdot\left\|x_{n+1}-x_{n}\right\|<M\left(\left(\frac{h(R)}{1-g(R)}\right)^{2 n}+\left(\frac{h(R)}{1-g(R)}\right)^{2 n-1}\right) \eta, \\
& \cdot\left\|F\left(x_{n+1}\right)\right\|<K(R+\eta+\delta)\left\|x_{n+1}-y_{n}\right\|,
\end{aligned}
$$


provided that $A_{i}=\left[x_{i}, x_{i}+F\left(x_{i}\right) ; F\right]$ is invertible and $y_{i}, x_{i}+F\left(x_{i}\right), x_{i+1} \in B\left(x_{0}, R\right)$, for all $i=1,2, \ldots, n$. In addition, it follows in the same way that $A_{n+1}^{-1}$ exists,

$$
\begin{aligned}
& \left\|A_{n+1}^{-1}\right\| \leq \frac{\beta}{1-g(R)}, \\
& \left\|x_{n+1}+F\left(x_{n+1}\right)-x_{0}\right\|<(1+P) \eta+M h(R) \delta=R, \\
& \left\|y_{n+1}-x_{n+1}\right\|<M\left(\frac{h(R)}{1-g(R)}\right)^{2 n+1} \eta, \\
& \left\|y_{n+1}-x_{0}\right\|<(1+P) \eta<R, \\
& \left\|F\left(y_{n+1}\right)\right\|<K(\eta+\delta)\left\|y_{n+1}-x_{n+1}\right\|, \\
& \left\|x_{n+2}-y_{n+1}\right\|<M\left(\frac{h(R)}{1-g(R)}\right)^{2 n+2} \eta, \\
& \left\|x_{n+2}-x_{n+1}\right\|<M\left(\left(\frac{h(R)}{1-g(R)}\right)^{2 n+2}+\left(\frac{h(R)}{1-g(R)}\right)^{2 n+1}\right) \eta, \\
& \left\|x_{n+2}-x_{0}\right\|<\left(1+M \sum_{j=0}^{2 n+2}\left(\frac{h(R)}{1-g(R)}\right)^{j}\right) \eta, \\
& \left\|F\left(x_{n+2}\right)\right\|<K(R+\eta+\delta)\left\|x_{n+2}-y_{n+1}\right\|,
\end{aligned}
$$

by (45), so that $y_{n+1}, x_{n+1}+F\left(x_{n+1}\right), x_{n+2} \in B\left(x_{0}, R\right)$ for all $n \in \mathbb{N}$.

Once we have seen that the sequence defined by method (42) is well-defined, we see that it is a Cauchy sequence. Indeed, since

$$
\left\|x_{n+i}-x_{n}\right\| \leq \sum_{j=1}^{i}\left\|x_{n+j}-x_{n+j-1}\right\|<\sum_{j=1}^{i} M\left(\left(\frac{h(R)}{1-g(R)}\right)^{2 n+2 j-2}+\left(\frac{h(R)}{1-g(R)}\right)^{2 n+2 j-3}\right) \eta
$$

for $i \geq 1$, and (44), it is clear that $\left\{x_{n}\right\}$ is a Cauchy sequence. As a consequence, the sequence $\left\{x_{n}\right\}$, given by (42), is convergent. Now, if $\lim _{n \rightarrow \infty} x_{n}=x^{*}$, it follows that $F\left(x^{*}\right)=0$ from the continuity of the function $F$, since

$$
\left\|F\left(x_{n}\right)\right\|<K(R+\eta+\delta)\left\|x_{n}-y_{n-1}\right\|
$$

and $\left\|x_{n}-y_{n-1}\right\| \rightarrow 0$, by letting $n \rightarrow \infty$.

Finally, we prove the uniqueness of the solution $x^{*}$ in $\overline{B\left(x_{0}, R\right)}$. Suppose that $y^{*}$ is another solution of $F(x)=0$ in $\overline{B\left(x_{0}, R\right)}$. If $A=\left[y^{*}, x^{*} ; F\right]$ is invertible, it follows that $x^{*}=y^{*}$, since $A\left(y^{*}-x^{*}\right)=F\left(y^{*}\right)-F\left(x^{*}\right)$. By the Banach lemma, we have that $A$ is invertible, since

$$
\left\|I-A_{0}^{-1} A\right\| \leq\left\|A_{0}^{-1}\right\|\left\|A_{0}-A\right\| \leq g(R)<1 .
$$

The proof is complete.

To finish this section, we analyse, under conditions (C1)-(C3), the semilocal convergence of the iterative method called frozen three-step Steffensen method, whose algorithm is:

$$
\left\{\begin{array}{l}
x_{0} \in D \\
y_{n}=x_{n}-\left[x_{n}, x_{n}+F\left(x_{n}\right) ; F\right]^{-1} F\left(x_{n}\right), \\
z_{n}=y_{n}-\left[x_{n}, x_{n}+F\left(x_{n}\right) ; F\right]^{-1} F\left(y_{n}\right), \\
x_{n+1}=z_{n}-\left[x_{n}, x_{n}+F\left(x_{n}\right) ; F\right]^{-1} F\left(z_{n}\right), \quad n \geq 0 .
\end{array}\right.
$$

Next, we present the new semilocal convergence result for (46).

We start with a technical lemma which is used later. The proof is immediate from algorithm (46) and the definition of the divided difference of first order. 
Lemma 5.2 Let $\left\{x_{n}\right\}$ be the sequence given by the frozen three-step Steffensen method given in (46). If $x_{n}, y_{n-1}, y_{n}, z_{n}, z_{n-1}$ are different points with $x_{n}, y_{n-1}, y_{n}, z_{n}, z_{n-1} \in D$, then

$$
\begin{aligned}
& F\left(y_{n}\right)=\left(\left[y_{n}, x_{n} ; F\right]-A_{n}\right)\left(y_{n}-x_{n}\right) \\
& F\left(z_{n}\right)=\left(\left[z_{n}, y_{n} ; F\right]-A_{n}\right)\left(z_{n}-y_{n}\right) \\
& F\left(x_{n}\right)=\left(\left[x_{n}, z_{n-1} ; F\right]-A_{n-1}\right)\left(x_{n}-z_{n-1}\right) .
\end{aligned}
$$

Theorem 5.3 Let $F: D \subseteq \mathbb{R}^{m} \longrightarrow \mathbb{R}^{m}$ be a nonlinear operator defined on a nonempty open convex domain D. Suppose that conditions (C1)-(C3) are satisfied. If the equation

$$
t=\frac{(1-g(t)) \eta}{1-2 g(t)}+g(t) \delta
$$

where $g(t)=K \beta(2 t+\delta)$ and $\eta=\beta \delta$, has at least one positive real root and the smallest positive real root, denoted by $R$, satisfies

$$
g(R)<1 / 2
$$

and $B\left(x_{0}, R\right) \subset D$, then the frozen three-step Steffensen method given in (46), starting at $x_{0}$, is well-defined and converges to a solution $x^{*}$ of $F(x)=0$. Moreover, the solution $x^{*}$ and the iterates $x_{n}$ belong to $\overline{B\left(x_{0}, R\right)}$ and $x^{*}$ is unique in $\overline{B\left(x_{0}, R\right)}$.

Proof. We begin by proving that the sequence given by method (46) is well-defined and $x_{n} \in B\left(x_{0}, R\right) \subset D$ for all $n \in \mathbb{N}$. From $(\mathrm{C} 1)-(\mathrm{C} 3)$, it follows that $y_{0}$ is well-defined and

$$
\left\|y_{0}-x_{0}\right\| \leq\left\|A_{0}^{-1}\right\|\left\|F\left(x_{0}\right)\right\| \leq \eta<R
$$

as a consequence of (47). Thus, $y_{0} \in B\left(x_{0}, R\right)$.

Moreover, from Lemma 5.2, it follows $F\left(y_{0}\right)=\left(\left[y_{0}, x_{0} ; F\right]-A_{0}\right)\left(y_{0}-x_{0}\right)$, so that

$$
\left\|F\left(y_{0}\right)\right\| \leq K\left(\left\|y_{0}-x_{0}\right\|+\left\|F\left(x_{0}\right)\right\|\right)\left\|y_{0}-x_{0}\right\|<g(R) \delta<\delta .
$$

Now, as $y_{0} \in B\left(x_{0}, R\right)$, it follows that $z_{0}$ is well-defined and

$$
\begin{gathered}
\left\|z_{0}-y_{0}\right\|<K \beta(R+\delta)\left\|y_{0}-x_{0}\right\|<g(R)\left\|y_{0}-x_{0}\right\| \leq \eta, \\
\left\|z_{0}-x_{0}\right\| \leq\left\|z_{0}-y_{0}\right\|+\left\|y_{0}-x_{0}\right\|<\left(1+\frac{g(R)}{1-g(R)}\right)\left\|y_{0}-x_{0}\right\|<\frac{1-g(R)}{1-2 g(R)} \eta<R, \\
\left\|F\left(z_{0}\right)\right\| \leq K\left(\left\|z_{0}-x_{0}\right\|+\left\|y_{0}-x_{0}\right\|+\left\|F\left(x_{0}\right)\right\|\right)\left\|z_{0}-y_{0}\right\|<g(R) \delta<\delta .
\end{gathered}
$$

As $z_{0} \in B\left(x_{0}, R\right)$, it follows that $x_{1}$ is well-defined and $x_{1} \in B\left(x_{0}, R\right)$, since

$$
\begin{gathered}
\left\|x_{1}-z_{0}\right\|<g(R)\left\|z_{0}-y_{0}\right\|<\left(\frac{g(R)}{1-g(R)}\right)^{2}\left\|y_{0}-x_{0}\right\|<\eta<R . \\
\left\|x_{1}-x_{0}\right\| \leq\left\|x_{1}-z_{0}\right\|+\left\|z_{0}-x_{0}\right\|<\left(1+\frac{g(R)}{1-g(R)}+\left(\frac{g(R)}{1-g(R)}\right)^{2}\right)\left\|y_{0}-x_{0}\right\|<\frac{1-g(R)}{1-2 g(R)} \eta<R .
\end{gathered}
$$

In addition, as $F\left(x_{1}\right)=\left(\left[x_{1}, z_{0} ; F\right]-A_{0}\right)\left(x_{1}-z_{0}\right)$, it follows

$$
\left\|F\left(x_{1}\right)\right\|<K(2 R+\delta)\left\|x_{1}-z_{0}\right\|<g(R) \delta<\delta
$$

and

$$
\left\|x_{1}+F\left(x_{1}\right)-x_{0}\right\| \leq\left\|x_{1}-x_{0}\right\|+\left\|F\left(x_{1}\right)\right\|<\frac{1-g(R)}{1-2 g(R)} \eta+g(R) \delta=R,
$$

so that $x_{1}+F\left(x_{1}\right) \in B\left(x_{0}, R\right)$. 
Now, we prove the existence of the operator $A_{1}^{-1}$. By (C3), we see that $\left\|I-A_{0}^{-1} A_{1}\right\| \leq g(R)<1$ and, by the Banach lemma, the operator $A_{1}^{-1}$ exists and is such that

$$
\left\|A_{1}^{-1}\right\| \leq \frac{\beta}{1-g(R)} .
$$

Taking into account the previous ideas, we obtain easily

$$
\begin{gathered}
\left\|y_{1}-x_{1}\right\|<\frac{g(R)}{1-g(R)}\left\|x_{1}-z_{0}\right\|<\left(\frac{g(R)}{1-g(R)}\right)^{3}\left\|y_{0}-x_{0}\right\|<\eta<R, \\
\left\|y_{1}-x_{0}\right\| \leq\left\|y_{1}-x_{1}\right\|+\left\|x_{1}-x_{0}\right\|<\left(\sum_{i=0}^{3}\left(\frac{g(R)}{1-g(R)}\right)^{i}\right)\left\|y_{0}-x_{0}\right\|<\frac{1-g(R)}{1-2 g(R)} \eta<R, \\
\left\|F\left(y_{1}\right)\right\|<K(R+\delta)\left\|y_{1}-x_{1}\right\|<g(R) \delta<\delta .
\end{gathered}
$$

Similarly, it is easy to see

$$
\begin{gathered}
\left\|z_{1}-y_{1}\right\|<\frac{g(R)}{1-g(R)}\left\|y_{1}-x_{1}\right\|<\left(\frac{g(R)}{1-g(R)}\right)^{4}\left\|y_{0}-x_{0}\right\|<\eta<R, \\
\left\|z_{1}-x_{1}\right\| \leq\left\|z_{1}-y_{1}\right\|+\left\|y_{1}-x_{1}\right\|<\left(\left(\frac{g(R)}{1-g(R)}\right)^{4}+\left(\frac{g(R)}{1-g(R)}\right)^{3}\right)\left\|y_{0}-x_{0}\right\|<\frac{1-g(R)}{1-2 g(R)} \eta<R, \\
\left\|z_{1}-x_{0}\right\| \leq\left\|z_{1}-x_{1}\right\|+\left\|x_{1}-x_{0}\right\|<\left(\sum_{i=0}^{4}\left(\frac{g(R)}{1-g(R)}\right)^{i}\right)\left\|y_{0}-x_{0}\right\|<\frac{1-g(R)}{1-2 g(R)} \eta<R, \\
\left\|F\left(z_{1}\right)\right\|<K(2 R+\delta)\left\|z_{1}-y_{1}\right\|<g(R) \delta<\delta .
\end{gathered}
$$

Besides, it follows

$$
\begin{gathered}
\left\|x_{2}-z_{1}\right\|<\frac{g(R)}{1-g(R)}\left\|z_{1}-y_{1}\right\|<\left(\frac{g(R)}{1-g(R)}\right)^{5}\left\|y_{0}-x_{0}\right\|<\eta<R, \\
\left\|x_{2}-x_{1}\right\| \leq\left\|x_{2}-z_{1}\right\|+\left\|z_{1}-x_{1}\right\|<\left(\sum_{i=3}^{5}\left(\frac{g(R)}{1-g(R)}\right)^{i}\right)\left\|y_{0}-x_{0}\right\|<\frac{1-g(R)}{1-2 g(R)} \eta<R, \\
\left\|x_{2}-x_{0}\right\| \leq\left\|x_{2}-z_{1}\right\|+\left\|z_{1}-x_{0}\right\|<\left(\sum_{i=0}^{5}\left(\frac{g(R)}{1-g(R)}\right)^{i}\right)\left\|y_{0}-x_{0}\right\|<\frac{1-g(R)}{1-2 g(R)} \eta<R .
\end{gathered}
$$

Therefore, $x_{2} \in B\left(x_{0}, R\right)$. In addition, from (48), we have

$$
\left\|F\left(x_{2}\right)\right\|<K(2 R+\delta)\left\|x_{2}-z_{1}\right\|<g(R) \delta<\delta,
$$

so that

$$
\left\|x_{2}+F\left(x_{2}\right)-x_{0}\right\| \leq\left\|x_{2}-x_{0}\right\|+\left\|F\left(x_{2}\right)\right\|<\frac{1-g(R)}{1-2 g(R)} \eta+g(R) \delta=R
$$

and $x_{2}+F\left(x_{2}\right) \in B\left(x_{0}, R\right)$.

Now, following an argument similar to the one developed previously and applying an inductive procedure, it is easy to see the following recurrence relations:

The operator $A_{n}^{-1}$ exists and is such that $\left\|A_{n}^{-1}\right\| \leq \frac{\beta}{1-g(R)}$,

$$
\left\|y_{n}-x_{n}\right\|<\left(\frac{g(R)}{1-g(R)}\right)^{3 n}\left\|y_{0}-x_{0}\right\|<\eta<R,
$$




$$
\begin{aligned}
& \left\|y_{n}-x_{0}\right\|<\left(\sum_{i=0}^{3 n}\left(\frac{g(R)}{1-g(R)}\right)^{i}\right)\left\|y_{0}-x_{0}\right\|<\frac{1-g(R)}{1-2 g(R)} \eta<R, \\
& \left\|F\left(y_{n}\right)\right\|<K(R+\delta)\left\|y_{n}-x_{n}\right\|<g(R) \delta<\delta, \\
& \left\|z_{n}-y_{n}\right\|<\left(\frac{g(R)}{1-g(R)}\right)^{3 n+1}\left\|y_{0}-x_{0}\right\|<\eta<R, \\
& \left\|z_{n}-x_{n}\right\|<\left(\left(\frac{g(R)}{1-g(R)}\right)^{3 n+1}+\left(\frac{g(R)}{1-g(R)}\right)^{3 n}\right)\left\|y_{0}-x_{0}\right\|<R, \\
& \left\|z_{n}-x_{0}\right\|<\left(\sum_{i=0}^{3 n+1}\left(\frac{g(R)}{1-g(R)}\right)^{i}\right)\left\|y_{0}-x_{0}\right\|<\frac{1-g(R)}{1-2 g(R)} \eta<R, \\
& \left\|F\left(z_{n}\right)\right\|<K(2 R+\delta)\left\|z_{n}-y_{n}\right\|<g(R) \delta<\delta, \\
& \left\|x_{n+1}-z_{n}\right\|<\frac{g(R)}{1-g(R)}\left\|z_{n}-y_{n}\right\|<\left(\frac{g(R)}{1-g(R)}\right)^{3 n+2}\left\|y_{0}-x_{0}\right\|<\eta<R, \\
& \left\|x_{n+1}-x_{n}\right\|<\left(\sum_{i=3 n}^{3 n+2}\left(\frac{g(R)}{1-g(R)}\right)^{i}\right)\left\|y_{0}-x_{0}\right\|<\frac{1-g(R)}{1-2 g(R)} \eta<R, \\
& \left\|x_{n+1}-x_{0}\right\|<\left(\sum_{i=0}^{3 n+2}\left(\frac{g(R)}{1-g(R)}\right)^{i}\right)\left\|y_{0}-x_{0}\right\|<\frac{1-g(R)}{1-2 g(R)} \eta<R, \\
& \left\|F\left(x_{n+1}\right)\right\|<K(2 R+\delta)\left\|x_{n+1}-z_{n}\right\|<g(R) \delta<\delta, \\
& \left\|x_{n+1}+F\left(x_{n+1}\right)-x_{0}\right\|<\frac{1-g(R)}{1-2 g(R)} \eta+g(R) \delta=R .
\end{aligned}
$$

As a consequence, $y_{n}, z_{n}, x_{n+1}, x_{n+1}+F\left(x_{n+1}\right) \in B\left(x_{0}, R\right)$ for all $n \in \mathbb{N}$.

After that, we continue by proving that the sequence given by (46) is well-defined and $x_{n} \in B\left(x_{0}, R\right) \subset D$ for all $n \in \mathbb{N}$. For this, it is sufficient to apply the recurrence relations previously obtained. Then, we see that the sequence given by (46) is a Cauchy sequence. Indeed, since

$$
\left\|x_{n+i}-x_{n}\right\| \leq \sum_{j=1}^{i}\left\|x_{n+j}-x_{n+j-1}\right\|<\sum_{j=1}^{i}\left(\sum_{i=3 n+3 j-3}^{3 n+3 j-1}\left(\frac{g(R)}{1-g(R)}\right)^{i}\right)\left\|y_{0}-x_{0}\right\|,
$$

for $i \geq 1$, and (48), it is clear that $\left\{x_{n}\right\}$ is a Cauchy sequence. As a consequence, $\left\{x_{n}\right\}$ is convergent. Now, if $\lim _{n \rightarrow \infty} x_{n}=x^{*}$, it follows that $F\left(x^{*}\right)=0$ from the continuity of the function $F$, since

$$
\left\|F\left(x_{n}\right)\right\|<K(2 R+\delta)\left\|x_{n}-z_{n-1}\right\|
$$

and $\left\|x_{n}-z_{n-1}\right\| \| \rightarrow 0$ by letting $n \rightarrow \infty$.

Finally, we prove the uniqueness of the solution $x^{*}$ in $\overline{B\left(x_{0}, R\right)}$. Suppose that $y^{*}$ is another solution of $F(x)=0$ in $\overline{B\left(x_{0}, R\right)}$. If $A=\left[y^{*}, x^{*} ; F\right]$ is invertible, it follows that $x^{*}=y^{*}$, since $A\left(y^{*}-x^{*}\right)=F\left(y^{*}\right)-F\left(x^{*}\right)$. By the Banach lemma, we have that $A$ is invertible, since

$$
\left\|I-A_{0}^{-1} A\right\| \leq\left\|A_{0}^{-1}\right\|\left\|A_{0}-A\right\| \leq g(R)<1 .
$$

The proof is complete. 


\section{Application}

Now, we consider nonlinear integral equations of mixed Hammerstein type of the form

$$
x(s)=f(s)+\int_{a}^{b} G(s, t) H(t, x(t)) d t, \quad s \in[a, b],
$$

where $-\infty<a<b<+\infty, f, G$ y $H$ are known functions and $x$ is a solution to be determined. Integral equations of this type appear very often in several applications to real world problems. For example, in problems of dynamic models of chemical reactors [5], vehicular traffic theory, biology and queuing theory [6]. The Hammerstein integral equations also appear in the electro-magnetic fluid dynamics and can be reformulated as two-point boundary value problems with certain nonlinear boundary conditions and in multidimensional analogues which appear as reformulations of elliptic partial differentiable equations with nonlinear boundary conditions (see [15] and the references given there).

Solving equation (49) is equivalent to solving $\mathcal{F}(x)=0$, where $\mathcal{F}: D \subset \mathcal{C}[a, b] \longrightarrow \mathcal{C}[a, b]$ and

$$
[\mathcal{F}(x)](s)=x(s)-f(s)-\int_{a}^{b} G(s, t) H(t, x(t)) d t, \quad s \in[a, b] .
$$

If we consider (49) where $G$ is the Green function in $[a, b] \times[a, b]$, we then use a discretization process to transform equation (49) into a finite dimensional problem by approximating the integral of (49) by a GaussLegendre quadrature formula with $m$ nodes:

$$
\int_{a}^{b} q(t) d t \simeq \sum_{i=1}^{m} w_{i} q\left(t_{i}\right)
$$

where the nodes $t_{i}$ and the weights $w_{i}$ are determined.

If we denote the approximations of $x\left(t_{i}\right)$ and $f\left(t_{i}\right)$ by $x_{i}$ and $f_{i}$, respectively, with $i=1,2, \ldots, m$, then equation (49) is equivalent to the following system of nonlinear equations:

where

$$
x_{i}=f_{i}+\sum_{j=1}^{m} a_{i j} H\left(t_{j}, x_{j}\right), \quad j=1,2, \ldots, m,
$$

$$
a_{i j}=w_{j} G\left(t_{i}, t_{j}\right)= \begin{cases}w_{j} \frac{\left(b-t_{i}\right)\left(t_{j}-a\right)}{b-a}, & j \leq i, \\ w_{j} \frac{\left(b-t_{j}\right)\left(t_{i}-a\right)}{b-a}, & j>i .\end{cases}
$$

Now, system (50) can be written as

$$
F(\mathbf{x}) \equiv \mathbf{x}-\mathbf{f}-A \mathbf{z}=0, \quad F: \mathbb{R}^{m} \longrightarrow \mathbb{R}^{m},
$$

where

$$
\begin{gathered}
\mathbf{x}=\left(x_{1}, x_{2}, \ldots, x_{m}\right)^{T}, \quad \mathbf{f}=\left(f_{1}, f_{2}, \ldots, f_{m}\right)^{T}, \quad A=\left(a_{i j}\right)_{i, j=1}^{m}, \\
\mathbf{z}=\left(H\left(t_{1}, x_{1}\right), H\left(t_{2}, x_{2}\right), \ldots, H\left(t_{m}, x_{m}\right)\right)^{T} .
\end{gathered}
$$

If we now consider that $\nu$ is the cost in products of evaluating the scalar function $H$, then we conclude that $\mu=\left(m^{2}+m \nu\right) / m=m+\nu$ for system (51). The cost of each method for this application is

$$
\mathcal{C}_{\Phi_{k}}(\nu, m, \ell)=\frac{m}{6}\left(8 m^{2}+3(2 \nu+6 k+3 \ell-1) m+6 k(\nu-1)+3(2 k-1) \ell\right), \quad \rho_{\Phi_{k}}=k+1 .
$$

In this application, when we compare the computational efficiency index $C E I_{k}$ of numerical methods $\Phi_{k}$, for $k=1,2,3$, the results are very similar to those of Theorem 4.1; namely, we have the same assertions but with a new value $\nu_{0}$, instead $\mu_{0}$, equal to

$$
\nu_{0}=\frac{1}{2} \frac{27 \log (3)-42 \log (2)+(11 \log (3)-18 \log (2)) \ell}{-5 \log (3)+8 \log (2)} \approx 5.28005-3.76002 \ell
$$


and $\nu_{0}>0$ for $\ell<\ell_{0} \approx 1.40426$.

Now, we consider a nonlinear integral equation of form (49). In particular,

$$
x(s)=1+\int_{0}^{1} G(s, t)\left(x(t)+\frac{1}{2} x(t)^{2}\right) d t, \quad s \in[0,1],
$$

where $x \in C[0,1], t \in[0,1]$, and the kernel $G$ is the Green function in $[0,1] \times[0,1]$. First, we write the integral equation as $\mathcal{F}(x)=0$, where $\mathcal{F}: C[0,1] \longrightarrow C[0,1]$ and

$$
\mathcal{F}(x)(s)=x(s)-1-\int_{0}^{1} G(s, t)\left(x(t)+\frac{1}{2} x(t)^{2}\right) d t, \quad s \in[0,1] .
$$

Next, as we have done before, we transform it into the following finite dimensional problem

$$
F(\mathbf{x}) \equiv \mathbf{x}-\mathbf{1}-A\left(\mathbf{x}+\frac{1}{2} \hat{\mathbf{x}}\right)=0, \quad F: \mathbb{R}^{m} \longrightarrow \mathbb{R}^{m},
$$

where $\mathbf{x}=\left(x_{1}, x_{2}, \ldots, x_{m}\right)^{T}, \mathbf{1}=(1,1, \ldots, 1)^{T}, A=\left(a_{i j}\right)_{i, j=1}^{m}$ and $\hat{\mathbf{x}}=\left(x_{1}^{2}, x_{2}^{2}, \ldots, x_{m}^{2}\right)^{T}$. For system (52), we have $\nu=1$ and obtain $C E I_{\Phi_{3}}>C E I_{\Phi_{2}}>C E I_{\Phi_{1}}$ for $m \geq 3$ and $C E I_{\Phi_{2}}>C E I_{\Phi_{3}}>C E I_{\Phi_{1}}$ for $m=2$ and $\ell<1.13831$.

After that, we choose $m=8$ and the starting point $\mathbf{x}_{\mathbf{0}}=\mathbf{1}$, and use methods (39), (42) and (46) to approximate a solution of (52). For this initial point, we obtain

Moreover,

$$
\delta=0.185338, \quad \beta=1.190068 \text { and } \quad K=0.0617795 .
$$

the solution of equation (40) is $R_{1}=0.255857 \ldots$ and $M+g\left(R_{1}\right)=0.0900<1$,

the solution of equation (43) is $R_{2}=0.249559 \ldots$ and $g\left(R_{2}\right)+h\left(R_{2}\right)=0.1092<1$,

the solution of equation (47) is $R_{3}=0.266861 \ldots$ and $g\left(R_{3}\right)=0.05765<1 / 2$.

Therefore, the hypotheses of Theorems 5.1, 5.2 and 5.3 are satisfied and guarantees that a unique solution of equation $F(x)=0$ exists in $B\left(x_{0}, R_{1}\right), B\left(x_{0}, R_{2}\right)$ and $B\left(x_{0}, R_{3}\right)$, respectively. In Table 1 we can see the numerical solution $\mathrm{x}^{*}=\left(x_{1}^{*}, x_{2}^{*}, \ldots, x_{8}^{*}\right)^{T}$ rounded to 25 decimal digits. In Tables $2-4$, we show the errors $\left\|\mathbf{e}_{\mathbf{n}}\right\|=\left\|\mathbf{x}^{*}-\mathbf{x}_{\mathbf{n}}\right\|$. Notice that the sequence vector $\left\{\mathbf{x}_{\mathbf{n}}\right\}$ is a good approximation of a solution of system (52) $\mathbf{x}^{*}$. See the sequences $\left\{\left\|F\left(\mathbf{x}_{\mathbf{n}}\right)\right\|\right\}$ in Tables $2-4$.

Table 1: Numerical solution $\mathbf{x}^{*}$ of system (52) rounded to 25 decimal digits

\begin{tabular}{|c|c|}
\hline \hline$i$ & $x_{i}^{*}$ \\
\hline 1,8 & 1.0182886072137290552534942 \\
2,7 & 1.0872823580289716117751721 \\
3,6 & 1.1763098552662677157367023 \\
4,5 & 1.2385164267408749447939584 \\
\hline \hline
\end{tabular}

The numerical computations were performed using the MPFR library of $\mathrm{C}++$ multi-precision arithmetics $[8,17]$ with 4096 digits of mantissa. All algorithms were compiled by g++(4.2.1) for i686-apple-darwin1 with libgmp (v.5.0.2) and libmpfr (v.3.1.0) libraries in a processor Intel囚 Xeon E5620, 2.4GHz (64-bit machine). In this machine the quotient and product ratio is $\ell=1.731$.

The following classical stopping criterium

$$
\left\|e_{I}\right\|=\left\|x_{I}-\alpha\right\|>10^{-\nu} \quad \text { and } \quad\left\|e_{I+1}\right\|<10^{-\nu}, \text { where } \quad \nu=4096,
$$

is replaced by

$$
\left\|\breve{e}_{I}\right\|>10^{-\eta} \text { and }\left\|\breve{e}_{I+1}\right\|<10^{-\eta} \text {, where } \eta=[\nu(\rho-1) / \rho] \text {, }
$$


Table 2: Absolute errors obtained by method (39) and $\left\|F\left(\mathbf{x}_{\mathbf{n}}\right)\right\|$ for system (52)

\begin{tabular}{r|c|c|}
\hline \hline$n$ & $\left\|\mathbf{e}_{\mathbf{n}}\right\|$ & $\left\|F\left(\mathbf{x}_{\mathbf{n}}\right)\right\|$ \\
\hline 0 & $2.38 \mathrm{e}-0001$ & $1.85 \mathrm{e}-0001$ \\
1 & $6.05 \mathrm{e}-0003$ & $4.67 \mathrm{e}-0003$ \\
2 & $3.86 \mathrm{e}-0006$ & $2.99 \mathrm{e}-0006$ \\
3 & $1.57 \mathrm{e}-0012$ & $1.21 \mathrm{e}-0012$ \\
4 & $2.59 \mathrm{e}-0025$ & $2.00 \mathrm{e}-0025$ \\
5 & $7.04 \mathrm{e}-0051$ & $5.44 \mathrm{e}-0051$ \\
6 & $5.21 \mathrm{e}-0102$ & $4.02 \mathrm{e}-0102$ \\
7 & $2.85 \mathrm{e}-0204$ & $2.20 \mathrm{e}-0204$ \\
8 & $8.51 \mathrm{e}-0409$ & $6.58 \mathrm{e}-0409$ \\
9 & $7.60 \mathrm{e}-0818$ & $5.88 \mathrm{e}-0818$ \\
10 & $6.07 \mathrm{e}-1636$ & $4.69 \mathrm{e}-1636$ \\
11 & $3.87 \mathrm{e}-3272$ & $2.99 \mathrm{e}-3272$ \\
\hline \hline
\end{tabular}

Table 3: Absolute errors obtained by method (42) and $\left\|F\left(\mathbf{x}_{\mathbf{n}}\right)\right\|$ for system (52)

\begin{tabular}{|c|c|c|}
\hline \hline$n$ & $\left\|\mathbf{e}_{\mathbf{n}}\right\|$ & $\left\|F\left(\mathbf{x}_{\mathbf{n}}\right)\right\|$ \\
\hline 0 & $2.38 \mathrm{e}-0001$ & $1.85 \mathrm{e}-0001$ \\
1 & $2.32 \mathrm{e}-0004$ & $1.79 \mathrm{e}-0004$ \\
2 & $2.18 \mathrm{e}-0013$ & $1.69 \mathrm{e}-0013$ \\
3 & $1.79 \mathrm{e}-0040$ & $1.39 \mathrm{e}-0040$ \\
4 & $9.98 \mathrm{e}-0122$ & $7.71 \mathrm{e}-0122$ \\
5 & $1.72 \mathrm{e}-0365$ & $1.33 \mathrm{e}-0365$ \\
6 & $8.71 \mathrm{e}-1097$ & $6.73 \mathrm{e}-1097$ \\
7 & $1.14 \mathrm{e}-3290$ & $8.82 \mathrm{e}-3291$ \\
\hline \hline
\end{tabular}

Table 4: Absolute errors obtained by method (46) and $\left\|F\left(\mathbf{x}_{\mathbf{n}}\right)\right\|$ for system (52)

\begin{tabular}{|c|c|c|}
\hline \hline$n$ & $\left\|\mathbf{e}_{\mathbf{n}}\right\|$ & $\left\|F\left(\mathbf{x}_{\mathbf{n}}\right)\right\|$ \\
\hline 0 & $2.38 \mathrm{e}-0001$ & $1.85 \mathrm{e}-0001$ \\
1 & $9.00 \mathrm{e}-0006$ & $6.95 \mathrm{e}-0006$ \\
2 & $1.88 \mathrm{e}-0023$ & $1.45 \mathrm{e}-0023$ \\
3 & $3.52 \mathrm{e}-0094$ & $2.72 \mathrm{e}-0094$ \\
4 & $4.34 \mathrm{e}-0377$ & $3.36 \mathrm{e}-0377$ \\
5 & $1.01 \mathrm{e}-1508$ & $7.80 \mathrm{e}-1509$ \\
\hline \hline
\end{tabular}

where the infinity norm is used (see [11]). Moreover, $\breve{e}_{n}$ is obtained by

$$
\breve{e}_{n}=\left(\frac{F_{r}\left(x_{n}\right)}{F_{r}\left(x_{n-1}\right)}\right)_{1 \leq r \leq m} .
$$

Note that this criterion is independent of the knowledge of the root.

To evaluate the numerical efficiency of each method, we compute the factor $\tilde{\kappa}$ (see [10]); that is, for each iteration $n$ we get the precision (number of correct decimals) $D_{n}$ in computational time $\widetilde{\Theta}\left(D_{n}\right)$ (elapsed time 
from $D_{0}$ to obtain $D_{n}$ correct decimals), where

$$
D_{n} \approx-\log _{10}\left\|e_{n}\right\| \approx-\frac{\rho}{\rho-1} \log _{10}\left\|\breve{e}_{n}\right\| .
$$

We approximate the pairs $\left(\log D_{n}, \widetilde{\Theta}\left(D_{n}\right)\right), 1 \leq n \leq I$, in the sense of least-squares, by a polynomial of degree one, where the slope $\tilde{\kappa}$ is computed; namely, $\tilde{\kappa}$ is a coefficient that measures the time of execution in function of the approximate number of correct decimals. That is:

$$
\widetilde{\Theta}\left(D_{I}\right)=\tilde{\kappa}\left(\log D_{I}-\log D_{0}\right) .
$$

Taking into account this slope, we compare the time factor $T F$ with the computed time factor $\widetilde{T F}$ defined by

$$
\widetilde{T F}=\frac{\widetilde{\Theta}\left(D_{I}\right)}{t_{p} \log q}=\frac{\tilde{\kappa}}{t_{p}} \approx T F=\frac{1}{\log C E I},
$$

where $t_{p}$ is the necessary time spent by one product and $q=D_{I} / D_{0}$.

In these experiments we calculate the computational order of convergence $\breve{\rho}$, called PCLOC and defined in [11], by

$$
\breve{\rho}=\frac{\log \left\|F\left(x_{I}\right)\right\|}{\log \left\|F\left(x_{I-1}\right)\right\|} .
$$

If $\rho=\breve{\rho} \pm \Delta \breve{\rho}$, where $\rho$ is the local order of convergence and $\Delta \breve{\rho}$ is the error of PCLOC, then we get $\Delta \breve{\rho}<10^{-2}$. This fact means that, in all computations of PCLOC, we obtain at least three significant digits and this result is a good test of the local order of convergence of the iterative family presented in this paper.

Table 5 shows the results obtained for the iterative methods $\Phi_{k}, 1 \leq k \leq 3$. It is shown the measure time factor $T F$ defined in $(56)$, the necessary time $\widetilde{\Theta}\left(D_{I}\right)$ in miliseconds to reach the $I$-iteration, the number $D_{I}$ of correct decimals in $x_{I}$, the slope $\tilde{\kappa}$ and the computed time factor $\widetilde{T F}$. Furthermore, the last column shows the percentage of relative error $r_{T F}$ between $T F$ and $\widetilde{T F}$.

Table 5: Numerical results for the non linear system (52).

\begin{tabular}{|cccccccc|}
\hline \hline & $T F$ & $I$ & $\widetilde{\Theta}\left(D_{I}\right)$ & $D_{I}$ & $\tilde{\kappa}$ & $\widetilde{T F}$ & $r_{T F}$ \\
\hline$\Phi_{1}$ & 3379 & 11 & 169.46 & 3271 & 51.52 & 3434 & 1.65 \\
$\Phi_{2}$ & 2429 & 7 & 124.22 & 3290 & 37.35 & 2490 & 2.51 \\
$\Phi_{3}$ & 2161 & 5 & 97.50 & 1508 & 32.95 & 2197 & 1.66 \\
\hline \hline
\end{tabular}

Notice that the preceding results agree with the theoretical ones exposed previously. Observing the computed time factor $\widetilde{T F}$ of the different methods, we conclude that they also agree with the computational efficiency index $C E I$ or the time factor $T F$. Moreover, the inequalities

$$
C E I_{3}>C E I_{2}>C E I_{1},
$$

are in concordance with those of Theorem 4.1.

\section{Concluding remarks}

We present, in this paper, a generalization of a family of Steffensen-type methods with three frozen steps for solving nonlinear equations. A local convergence analysis of this family is given, where three specific divided difference operators are considered and studied in detail. Besides, we obtain four families of iterative methods with optimal local order of convergence. 
Also, new variants of the families are constructed and a study of their computational efficiencies is carried out. We also analyze the semilocal convergence of these variants. Moreover, an application of a nonlinear integral equation of mixed Hammerstein type is presented, where the use of multiple precision and a stopping criterion are implemented without using any known root.

Finally, where we compare the orders, efficiencies and elapsed times of the suggested methods that support the theoretical results claimed. We point out that the real efficiency of each method is related to the slope of the lines in the sense of least-squares.

\section{References}

[1] S. Amat, S. Busquier, V. Candela, A class of quasi-Newton generalized Steffensen methods on Banach spaces, J. Comput. Appl. Math. 149 (2002) 397-406.

[2] S. Amat, S. Busquier, J.M. Gutiérrez, Third-order iterative methods with applications to Hammerstein equations: a unified approach, J. Comput. Appl. Math. 235 (2011) 2936-2943.

[3] I.K. Argyros, J.A. Ezquerro, J.M. Gutiérrez, M.A. Hernández, S. Hilout, On the semilocal convergence of efficient Chebyshev-Secant-type methods, J. Comput. Appl. Math. 235 (2011) 3195-3206.

[4] I.K. Argyros, H. Ren, Efficient Steffensen-type algorithms for solving nonlinear equations, Int. J. Comput. Math. 90 (2013) 691-704.

[5] D.D. Bruns, J.E. Bailey, Nonlinear feedback control for operating a nonisothermal CSTR near an unstable steady state, Chem. Eng. Sci. 32 (1977), 257-264.

[6] K. Deimling, Nonlinear functional analysis, Springer-Verlag, Berlin, 1985.

[7] J.A. Ezquerro, M.A. Hernández, Increasing the applicability of Steffensen's method, J. Math. Anal. Appl. 418, 2 (2014) 1062-1073.

[8] L. Fousse, G. Hanrot, V. Lefèvre, P. Pélissier and P. Zimmermann, MPFR: a multiple-precision binary floating-point library with correct rounding, ACM Trans. Math. Softw. 33, 2 (2007) Art. 13 (15 pp.)

[9] M. Grau-Sánchez, A. Grau, M. Noguera, Frozen divided difference scheme for solving systems of nonlinear equations, J. Comput. Appl. Math. 235 (2011) 1739-1743.

[10] M. Grau-Sánchez, M. Noguera, A technique to choose the most efficient method between secant method and some variants, Appl. Math. Comput. 218 (2012) 6415-6426.

[11] M. Grau-Sánchez, A. Grau, M. Noguera, J.R. Herrero, A study on new computational local orders of convergence, Appl. Math. Lett. 25 (2012) 2023-2030.

[12] M. Grau-Sánchez, M. Noguera, J.L. Diaz-Barrero, On the local convergence of a family of two-step iterative methods for solving nonlinear equations, J. Comput. Appl. Math. 255 (2014) 753-764.

[13] A.M. Ostrowski, Solutions of Equations and System of Equations, Academic Press, New York, 1960.

[14] F.A. Potra, V. Pták, Nondiscrete induction and iterative methods, Pitman Publishing Limited, London, 1984 .

[15] J. Rashidinia, M. Zarebnia, New approach for numerical solution of Hammerstein integral equations, Appl. Math. Comput. 185 (2007), 147-154.

[16] I.F. Steffensen, Remarks on iteration, Skand. Aktuarietidskr. 16 (1933) 64-72.

[17] The GNU MPFR library 3.1.0. Available in http://www.mpfr.org

[18] J.F. Traub, Iterative methods for the solution of equations, Prentice-Hall, Englewood Cliffs, New Jersey, 1964 . 